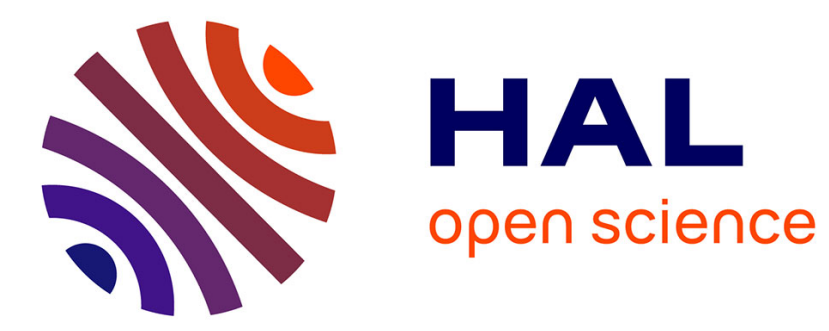

\title{
Effet de pic structural et autostabilité dans un alliage $\mathrm{Pb}-\mathrm{In}-\mathrm{Na}$
}

O. Bethoux, G. Schumacher

\section{To cite this version:}

O. Bethoux, G. Schumacher. Effet de pic structural et autostabilité dans un alliage Pb-In-Na. Revue de Physique Appliquée, 1973, 8 (4), pp.439-441. 10.1051/rphysap:0197300804043900 . jpa-00243711

\section{HAL Id: jpa-00243711 https://hal.science/jpa-00243711}

Submitted on 1 Jan 1973

HAL is a multi-disciplinary open access archive for the deposit and dissemination of scientific research documents, whether they are published or not. The documents may come from teaching and research institutions in France or abroad, or from public or private research centers.
L'archive ouverte pluridisciplinaire HAL, est destinée au dépôt et à la diffusion de documents scientifiques de niveau recherche, publiés ou non, émanant des établissements d'enseignement et de recherche français ou étrangers, des laboratoires publics ou privés. 


\title{
EFFET DE PIC STRUCTURAL ET AUTOSTABILITÉ DANS UN ALLIAGE Pb-In-Na
}

\section{O. BETHOUX et G. SCHUMACHER}

CNRS, Centre de Recherches sur les Très Basses Températures, BP 166, 38042 Grenoble, Cedex, France

\begin{abstract}
Résumé. - Nous présentons des résultats préliminaires concernant un fort effet de pic dans l'hystérésis magnétique d'un alliage $\mathrm{Pb}-\mathrm{In}-\mathrm{Na}$, qui ouvrent des perspectives intéressantes quant à la possibilité d'obtenir des matériaux supraconducteurs autostables.

Abstract. - Preliminary results are presented concerning a peak effect in magnetic hysteresis of a $\mathrm{Pb}$-In-Na alloy. This opens up many interesting possibilities for obtaining autostable superconducting materials.
\end{abstract}

Une condition suffisante pour qu'un supraconducteur soit autostable est que la force d'ancrage des lignes de vortex augmente avec la température [1]-[2]. Nous présentons ici quelques résultats préliminaires obtenus avec un alliage $\mathrm{Pb}-\mathrm{In}-\mathrm{Na}$ où la condition $\mathrm{d} F_{\mathrm{p}} / \mathrm{d} T>0$ est associée à un fort « effet de pic » structural.

1. Echantillon et courbes d'aimantation. - L'échantillon dont on a mesuré l'aimantation (diamètre $5 \mathrm{~mm}$, longueur $15 \mathrm{~mm}$ ) est un alliage $\mathrm{Pb}$-In équi-atomique contenant $0,37 \%$ en poids de $\mathrm{Na}$ (soit $2,54 \%$ at). Préparé à partir de métaux purs $(99,999 \%)$, l'alliage a été fondu sous vide puis coulé en lingotière de cuivre refroidie à l'eau. L'échantillon de mesure a été recuit 11 jours à $200^{\circ} \mathrm{C}$, puis maintenu à la température ambiante; d'abord quelques jours, puis, après mesure de son aimantation à $4,2 \mathrm{~K}$, quatre semaines.

La figure 1 montre l'évolution de l'aimantation à $4,2 \mathrm{~K}$ avec la durée du maintien à la température ambiante (courbes $a$ et $b$ ). Les courbes d'aimantation présentent un fort «pic » de l'hystérésis magnétique traduisant un pic équivalent de la force d'ancrage des vortex. On peut noter que le pic grandit et se déplace vers les faibles champs lorsque la durée du revenu à la température ambiante augmente.

Bien que le diagramme d'équilibre du système ternaire $\mathrm{Pb}-\mathrm{In}$ - $\mathrm{Na}$ ne soit pas connu et que nous n'ayons pas encore effectué d'étude métallographique de l'alliage, le pic de l'aimantation peut être, à coup sûr, associé à la précipitation d'une phase plus riche en sodium que la solution solide initiale. En effet l'alliage $\mathrm{Pb}-\mathrm{In} 50 \%$ at est une solution solide possédant la structure cristalline du $\mathrm{Pb}$ (cfc) et dans l'alliage $\mathrm{Pb}-\mathrm{Na}$, le sodium précipite sous forme de $\mathrm{Pb}_{3} \mathrm{Na}$, sa limite de solubilité étant de $1,8 \%$ at à la température ambiante et $7 \%$ at à $200{ }^{\circ} \mathrm{C}$ [3]. Il est donc tout à fait probable que dans notre alliage, où la concentration en sodium

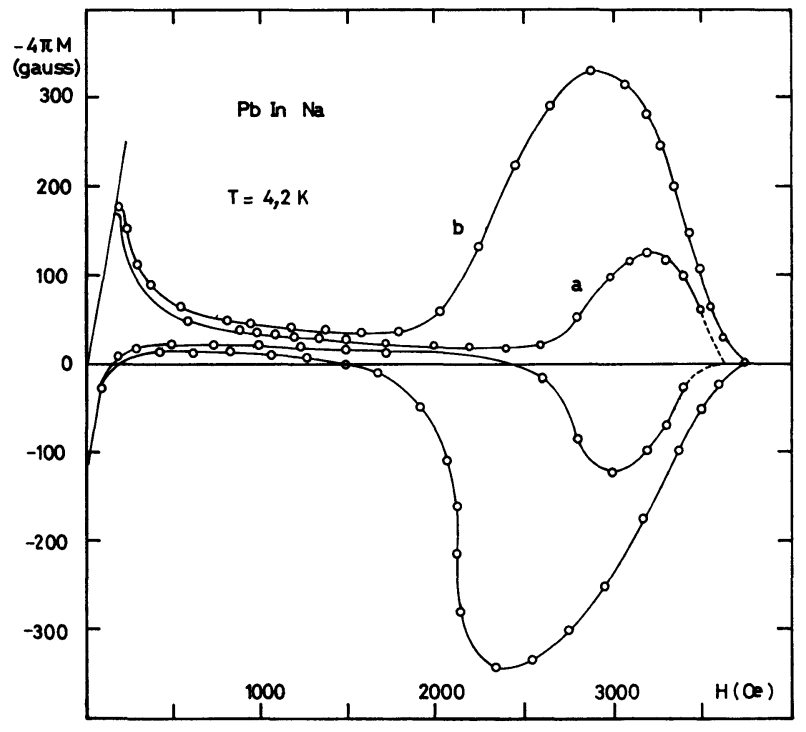

Fig. 1. - Aimantation en fonction du champ magnétique appliqué à $4,2 \mathrm{~K}$, de l'alliage $\left(\mathrm{Pb}_{0,5}-\mathrm{In}_{0,5}\right) \mathrm{Na} 2,54 \%$ at. Courbe $a$ : revenu de quelques jours à température ambiante. Courbe $b$ : revenu de quatre semaines à température ambiante.

est comprise entre ces deux valeurs, le sodium précipite aussi sous forme d'un composé $(\mathrm{Pb}, \mathrm{In})_{3} \mathrm{Na}$.

Par ailleurs, les faibles variations de $H_{\mathrm{c} 2}$ pendant la précipitation (quelques \% de variation par rapport au $H_{\mathrm{c} 2}$ de l'alliage $\mathrm{Pb}-\mathrm{In} 50 \%$ atomique) laissent supposer que la concentration de la solution solide a peu varié et donc que la phase précipitée est de composition très voisine de $\left(\mathrm{Pb}_{0,5} \mathrm{In}_{0,5}\right)_{3} \mathrm{Na}$.

Il apparaît donc que la variation d'amplitude et le déplacement du pic entre les courbes $a$ et $b$ sont liés au développement de la précipitation. D'après les résultats de Freyhardt [4] qui a étudié des alliages $\mathrm{Pb}-\mathrm{Na} 7 \%$ atomique, il est probable que l'évolution 
du pic est lié au grossissement des précipités et à la diminution corrélative de leur nombre.

Sur la figure 2 ont été reportées diverses courbes d'aimantation obtenues entre 4,2 et $6 \mathrm{~K}$ avec l'échantillon maintenu quatre semaines à la température ambiante. On peut voir que le pic se déplace en fonc-

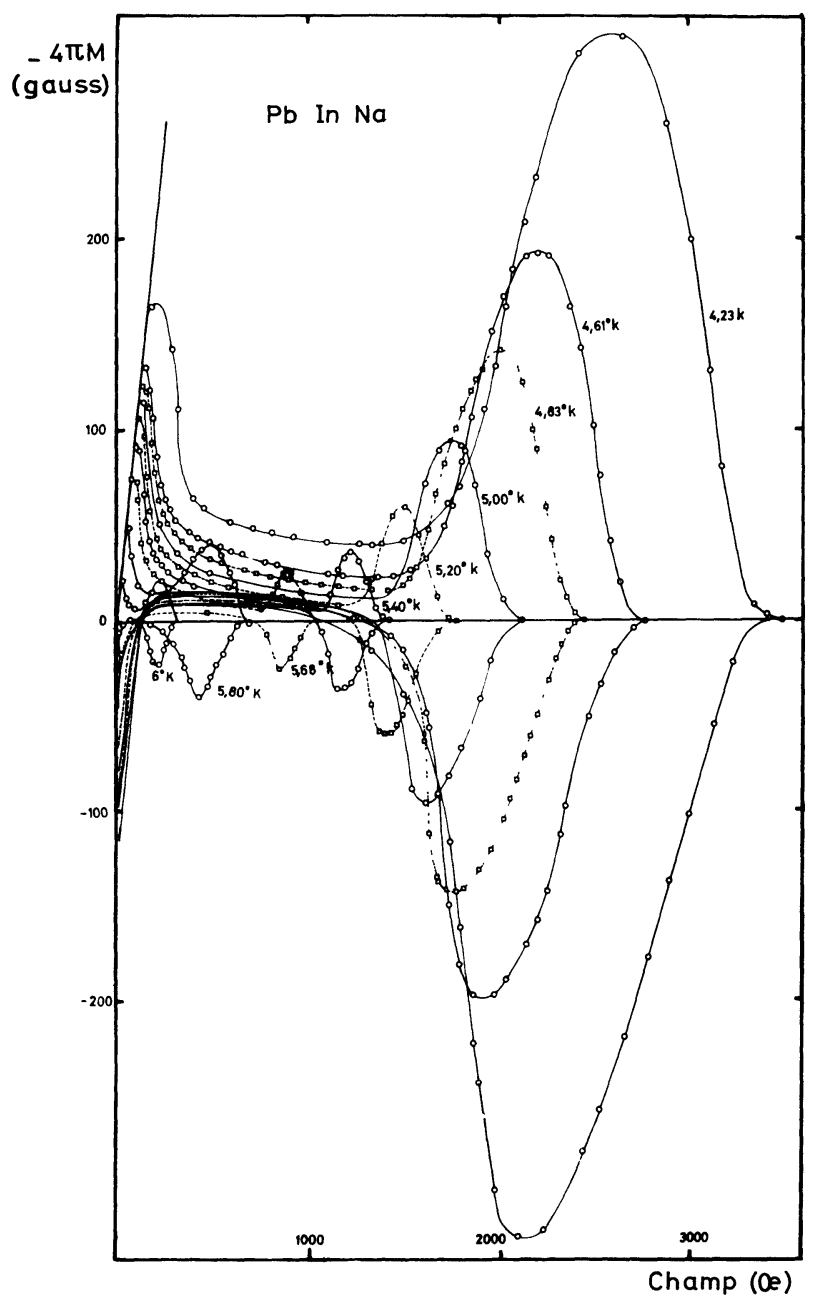

Fig. 2. - Aimantation en fonction du champ magnétique appliqué à différentes températures.

tion'de la température et que les courbes d'aimantation se coupent pour $H<2100$ Oe environ. Dans cette région de champ, il existe pour toute valeur de $H$, un intervalle de température dans lequel l'hystérésis magnétique, et donc la force d'ancrage, croissent avec la température. Cet effet est montré plus clairement sur la figure 3 pour $H=1500$ Oe. $\Delta T_{a}$ est l'intervalle de température où le matériau est autostable.

2. Mécanisme responsable de l'effet de pic. - Bien qu'une étude plus détaillée, structurale en particulier soit"nécessaire pour tenter de déterminer avec précision l'origine de l'effet de pic observé ici, les présents résultats peuvent être comparés aux modèles proposés dans la littérature. Ces modèles dont on pourra trouver une bonne revue dans la référence [5] sont $\mathrm{de}_{\mathrm{j}}^{-}$deux sortes; d'une part ceux qui supposent une variation rapide de la force d'interaction vortex-

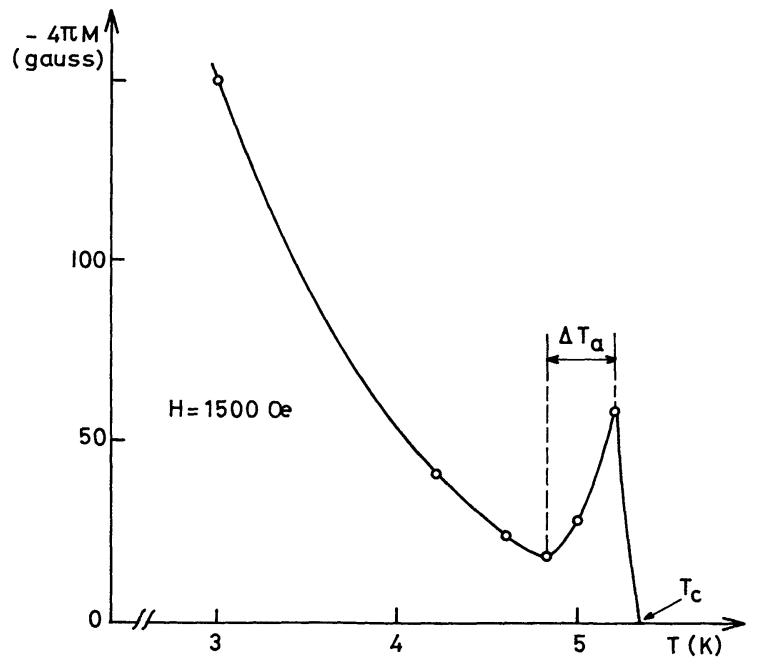

Fig. 3. - Aimantation dans un champ magnétique de $1500 \mathrm{Oe}$ en fonction de la température, $T_{\mathrm{c}}$ est la température critique pour le champ de $1500 \mathrm{Oe} ; \Delta T_{\mathrm{a}}$ est l'intervalle de température où l'alliage est autostable.

centres d'ancrage (transition supra-normale des précipités en particulier) d'autre part ceux qui supposent une variation du mode de sommation des forces d'interaction (effets de résonance, de synchronisation, etc...). Aucun des modèles de cette dernière catégorie ne permet d'expliquer nos résultats, en particulier les variations thermiques de l'amplitude et du champ correspondant au maximum du pic. Il semble donc qu'il s'agisse d'une variation rapide de la force d'interaction précipités-vortex. Compte tenu des résultats de Freyhardt [4] les précipités ont probablement des tailles de l'ordre de grandeur de $\xi(\xi(0) \sim 200 \AA)$, et les effets de proximité dus à la matrice supraconductrice doivent être importants. Dès lors, l'augmentation rapide de la force d'ancrage avec le champ magnétique peut être due à un mécanisme du type de celui décrit par Burger et Deutsher [6], ou, si $\kappa_{\mathrm{N}}(0)<1$, à une transition brutale des précipités au champ de basculement $H_{\mathbf{B}}$ [7] $\left(\kappa_{\mathrm{N}}(0)\right.$ est le paramètre de GinzburgLandau pour les précipités à l'interface précipitématrice). Effectivement le champ correspondant au pic, $H_{\mathrm{p}}$ varie bien qualitativement comme $H_{\mathrm{B}}$ [7] : il décroît lorsque la température augmente et la taille des particules normales augmente.

3. Conclusion. - Une étude expérimentale plus poussée de cet effet de pic est nécessaire pour tenter de vérifier les hypothèses avancées pour en expliquer l'origine. Si ces hypothèses s'avèrent exactes, on peut s'attendre à ce que ce mécanisme soit suffisamment général pour que l'on puisse le retrouver sur la plupart des solutions solides sursaturées pendant les premiers stades de la précipitation. En effet, il se trouvera toujours un moment où les précipités auront des dimensions de l'ordre de $\xi_{\mathrm{N}}$. Dès lors de très nombreux matériaux pourraient présenter des propriétés d'autostabilité, y compris les alliages de $\mathrm{Nb}$, les plus intéressants techniquement. 


\section{Bibliographie}

[1] Livingston, J. D., Appl. Phys. Lett. 8 (1966) 319.

[2] Hart, H. R. jr and Livingston, J. D., Proceedings LT 11. University of St-Andrews, 869 (1968).

[3] Hansen, M. H., Constitution of binary alloys (McGrawHill), 1958.

[4] FreyhardT, H., Thèse, Göttingen (1970).
[5] Campbell, A. M. and Evetts, J. E., Adv. Phys. 21 (1972) 90.

[6] Burger, J. P. et Deutscher, G., Revue Phys. Appl. 6 (1971) 505.

[7] Groupe d'Orsay sur la Supraconductivité, Phys. Kond. Mater. 6 (1967) 307. 